\title{
Queues with waiting time dependent service
}

\author{
R. Bekker • G.M. Koole • B.F. Nielsen • T.B. Nielsen
}

Received: 20 July 2009 / Revised: 20 March 2011 / Published online: 27 April 2011

(C) The Author(s) 2011. This article is published with open access at Springerlink.com

\begin{abstract}
Motivated by service levels in terms of the waiting-time distribution seen, for instance, in call centers, we consider two models for systems with a service discipline that depends on the waiting time. The first model deals with a single server that continuously adapts its service rate based on the waiting time of the first customer in line. In the second model, one queue is served by a primary server which is supplemented by a secondary server when the waiting of the first customer in line exceeds a threshold. Using level crossings for the waiting-time process of the first customer in line, we derive steady-state waiting-time distributions for both models. The results are illustrated with numerical examples.
\end{abstract}

Keywords Waiting-time distribution - Adaptive service rate $\cdot$ Call centers $\cdot$ Contact centers · Queues · Deterministic threshold · Overflow · Level crossing

Mathematics Subject Classification (2000) Primary 90B22 • 60K25 •

Secondary $68 \mathrm{M} 20 \cdot 60 \mathrm{~K} 30$

\section{Introduction}

In service systems, the tail probability (or distribution function) of the waiting time of customers is one of the main service-level indicators. For example, in call centers the service level is generally characterized by the telephone service factor (TSF), i.e., the

R. Bekker $(\bowtie) \cdot$ G.M. Koole

Dept. Mathematics, VU University Amsterdam, De Boelelaan 1081, 1081 HV, Amsterdam,

The Netherlands

e-mail: rbekker@few.vu.nl

B.F. Nielsen · T.B. Nielsen

Dept. Informatics and Mathematical Modelling, Technical University of Denmark, Richard Petersens Plads, 2800 Kgs. Lyngby, Denmark 
fraction of calls whose delay falls below a prespecified target. Typically, call centers use a $80-20$ TSF meaning that $80 \%$ of the calls should be taken into service within 20 seconds; see [14]. Motivated by performance measures in terms of tail probabilities of waiting times, we consider queueing systems where the service mechanism is based on waiting times of customers. This type of control policy is commonly used in call centers [21], and indeed the authors have often encountered it in various forms when working with call centers. However, the literature on it is limited. In the traditional queueing literature, routing and control are commonly based on the number of customers present.

The main goal of this paper is to find the steady-state waiting-time distribution for queueing systems where the service characteristics depend on the waiting time of the first customer in line. This type of service control seems to be new in the queueing literature, despite its widespread use in the industry. The aim of this paper is to show ways to analyze queueing models where the service mechanism depends on the waiting time. In what follows we use FIL as an abbreviation of first customer in line.

We consider two Markovian queueing models: (i) single-server queues with FIL waiting-time dependent service speed and (ii) a queue with two heterogeneous servers, where the secondary server is only activated as soon as the FIL waiting time exceeds some target level. For both models, the analysis is based on the waiting process of the first customer in line (FIL-process). Using level crossings, we find the steady-state distribution of the FIL-process and derive the waiting-time distribution as a corollary.

First, in Sect. 2, we study the single-server model, where the service speed can be continuously adapted based on the waiting time of the first customer in line. This model is related to the study of dams and queueing systems with workload-dependent service rates; see for example [4, 6, 16] or [25]. The difference is that the service speed here depends on the waiting time instead of the amount of work present.

Secondly, in Sect. 3, we consider a system with a single queue and two heterogeneous servers, where the secondary server takes the first customer in line into service as soon as his waiting time exceeds some threshold. The primary motivation for this model stems from routing mechanisms in call centers with operators in front and back offices. Typically, the only task of operators in the front office would be to answer calls whereas operators in the back office would have other assignments and only answer calls under high load. A common problem is then how to meet the service-level agreements while keeping the disturbance of the back office operators to a minimum; see [14] and references therein. Overflow problems are in general difficult to analyze, see [11], because the overflow traffic is not Poisson; the deterministic threshold of this model only adds to this. We believe though that the model is of independent interest and has its applications in other areas where the service level involves the (tail) distribution of the waiting time, as in, for example, telecommunication and production systems, or in supply chains with lead time decisions [20].

Related to the heterogeneous-servers model above is the slow-server problem; see $[18,19,24]$ and [26]. In the slow-server model, a single queue is served by two heterogeneous servers with service rates $\mu_{1}$ and $\mu_{2}$, where $\mu_{1}>\mu_{2}$. In [24] the author gives qualitative and explicit quantitative results on when to maintain or discard 
the slow server. In the models of [18] and [19], customers can be assigned to one of the servers depending on the number of customers present. There it was shown that the fast server should always be used and that the slow server should only be used if the number of customers exceeds some threshold. This result was derived for an infinite waiting space. We note that in case of a finite queue length, the optimal policy is not necessarily of a threshold type; see [26].

The literature on queueing models where the service time process depends on the waiting time is limited. In [3] a system with time dependent overflow is approximated by a queue-length dependent overflow. Prioritization based on adding different constants to the waiting times of customers is introduced in [17] and referred to as dynamic prioritization. There are some studies of single-server queues where the service time depends on the waiting time experienced by the customer in service (instead of the first customer in line); see [5, 23] and [27]. Furthermore, in [7] the authors consider an $\mathrm{M} / \mathrm{M} / 2$ queue where non-waiting customers receive a different rate of service than customers who first wait in line. Their analysis is based on the "system point method" [8], which is closely related to the level crossing equations [10] of Sect. 3.

Some numerical results are presented in Sect. 4. Conclusions and topics for further research can be found in Sect. 5 .

\section{Single-server queue}

In this section we consider a single-server queue where the service speed depends on the waiting time of the first customer in line. In particular, we assume that customers arrive according to a Poisson process with rate $\lambda$ and have exponentially distributed service requirements with mean $1 / \mu$. The service discipline is assumed to be FIFO. Denote by $W_{t}$ the waiting time of the first customer in the queue at time $t$, with the convention that $W_{t}=0$ if the queue is empty. Also, let $Y_{t}$ denote the number of customers in service at time $t$ (thus $Y_{t} \in\{0,1\}$ ). The service speed depends on the waiting time of the first customer in line and the service speed function is denoted by $r(\cdot)$. Let $r(0)$ be the service speed for state $\left(W_{t}, Y_{t}\right)=(0,1)$ and 0 be the speed for state $(0,0)$. For convenience, define $\rho_{0}=\lambda /(\mu r(0))$. We assume that $r(\cdot)$ is strictly positive, left-continuous, and has a strictly positive right limit on $(0, \infty)$.

The process $\left\{\left(W_{t}, Y_{t}\right), t \geq 0\right\}$ can now be described as follows. Given that $W_{t_{0}}=$ $w>0$ and the next service completion is at time $t_{1}>t_{0}$, the waiting-time process of the first customer in line during $\left(t_{0}, t_{1}\right)$ behaves as $W_{t_{0}+t}=w+t$ and $Y_{t_{0}+t}=1$. If $S_{w}$ denotes the time until the next service completion, conditioned on the initial waiting time $w>0$, then $\mathbb{P}\left(S_{w}>t\right)=\exp \left(-\mu \int_{w}^{w+t} r(y) \mathrm{d} y\right)$. At the moment of a service completion, the second customer in line (if there is any) becomes the first customer in line. Since the interarrival times between customers are exponentially distributed, we have

$$
W_{t_{1}^{+}}=\left(W_{t_{1}^{-}}-A_{\lambda}\right)^{+},
$$

where $(x)^{+}=\max \{x, 0\}$ and $A_{\lambda}$ denotes an exponential random variable of rate $\lambda$. 
It remains to specify the boundary cases of an empty queue. For $\left(0, Y_{t_{0}}\right)$, the time until the next state transition has an exponential distribution with rate $\lambda+\mu r(0) Y_{t_{0}}$. For $(0,1)$ the next state is $(0,0)$ with probability $\mu r(0) /(\lambda+\mu r(0))$, or $W_{t}$ starts to increase linearly, as described above, with probability $\lambda /(\lambda+\mu r(0))$. For $(0,0)$, the next state is $(0,1)$ with probability 1 .

Since the service requirements and interarrival times are exponentially distributed, the process $\left\{\left(W_{t}, Y_{t}\right), t \geq 0\right\}$ is a Markov process. Assuming that the system is stable (see [9, Corollary 4.2] for stability conditions), the process is regenerative and thus has a stationary distribution, see for example [2, Chap. VII]. Below, we determine the steady-state distribution of this process and derive from it the waiting-time distribution of an arbitrary customer. For this, we introduce the steady-state distribution of the FIL-process as $W^{\mathrm{FIL}}(x)=\lim _{t \rightarrow \infty} \mathbb{P}\left(W_{t} \leq x\right)$ and the corresponding density as $w^{\mathrm{FIL}}(x)=\mathrm{d} W^{\mathrm{FIL}}(x) / \mathrm{d} x$. For the atom at zero, $Y_{t}$ is included in the notation as $W^{\mathrm{FIL}}(0, y)=\lim _{t \rightarrow \infty} \mathbb{P}\left(W_{t}=0, Y_{t}=y\right)$.

Theorem 2.1 We have $W^{\mathrm{FIL}}(0,1)=\rho_{0} W^{\mathrm{FIL}}(0,0)$. The density of the FIL-process is

$$
w^{\mathrm{FIL}}(x)=\lambda \rho_{0} W^{\mathrm{FIL}}(0,0) \exp \left\{\int_{0}^{x}(\lambda-\mu r(y)) \mathrm{d} y\right\},
$$

where

$$
W^{\mathrm{FIL}}(0,0)=\left[1+\rho_{0}+\lambda \rho_{0} \int_{0}^{\infty} \exp \left\{\int_{0}^{x}(\lambda-\mu r(y)) \mathrm{d} y\right\} \mathrm{d} x\right]^{-1}
$$

It is instructive to derive the distribution of the FIL-process based on level crossing arguments. We refer to Remark 2.1 below for an alternative proof based on results in [6].

Proof For $x>0$, using (1), the level crossing equations read

$$
w^{\mathrm{FIL}}(x)=\int_{y=x}^{\infty} e^{-\lambda(y-x)} \mu r(y) w^{\mathrm{FIL}}(y) \mathrm{d} y .
$$

The left-hand side corresponds to upcrossings of level $x$ and the right-hand side corresponds to the long-run average number of downcrossings through level $x$. Observe that we have continuous upcrossings of waiting-time levels and downcrossings by jumps, where the jump sizes correspond to interarrival times between successive customers (in contrast to workloads in single-server queues). Taking derivatives on both sides of (2) yields

$$
\begin{aligned}
\frac{\mathrm{d}}{\mathrm{d} x} w^{\mathrm{FIL}}(x) & =\lambda\left[\int_{y=x}^{\infty} e^{-\lambda(y-x)} \mu r(y) w^{\mathrm{FIL}}(y) \mathrm{d} y\right]-\mu r(x) w^{\mathrm{FIL}}(x) \\
& =(\lambda-\mu r(x)) w^{\mathrm{FIL}}(x),
\end{aligned}
$$


where the second step follows from (2). The solution of this first-order differential equation can be readily obtained as

$$
w^{\mathrm{FIL}}(x)=C \exp \left\{\int_{0}^{x}(\lambda-\mu r(y)) \mathrm{d} y\right\} .
$$

Balancing the transitions between the interior part of the state space and the boundary part, we have

$$
\lambda W^{\mathrm{FIL}}(0,1)=\int_{0}^{\infty} e^{-\lambda y} \mu r(y) w^{\mathrm{FIL}}(y) \mathrm{d} y .
$$

Using the above and letting $x \downarrow 0$ in (2) yields $\lim _{x \downarrow 0} w^{\mathrm{FIL}}(x)=\lambda W^{\mathrm{FIL}}(0,1)$. Also, letting $x \downarrow 0$ in (3) determines the constant $C=\lim _{x \downarrow 0} w^{\mathrm{FIL}}(x)=\lambda W^{\mathrm{FIL}}(0,1)$.

Now, balancing the transitions between the two boundary states gives

$$
\lambda W^{\mathrm{FIL}}(0,0)=\mu r(0) W^{\mathrm{FIL}}(0,1),
$$

which enables us to determine the three constants in terms of $W^{\mathrm{FIL}}(0,0)$. Finally, using normalization, we have

$$
W^{\mathrm{FIL}}(0,0)+W^{\mathrm{FIL}}(0,1)+\lambda W^{\mathrm{FIL}}(0,1) \int_{0}^{\infty} \exp \left\{\int_{0}^{x}(\lambda-\mu r(y)) \mathrm{d} y\right\} \mathrm{d} x=1 .
$$

Expressing $W^{\mathrm{FIL}}(0,1)$ in $W^{\mathrm{FIL}}(0,0)$ and solving for $W^{\mathrm{FIL}}(0,0)$ completes the proof.

To determine the waiting time, we only need to consider the FIL-process at specific points in time. We introduce the waiting time an arbitrary customer experiences as $W$ and the distribution of this as $W(x)=\mathbb{P}(W \leq x)$. Using PASTA, it is easy to see that the atom at zero of the waiting time is given by $\mathbb{P}(W=0)=W^{\mathrm{FIL}}(0,0)$. In case of non-zero waiting times, the waiting times are given by the FIL-process embedded at epochs just before downward jumps.

Let $N_{s}(u, v)$ denote the number of customers taken into service during the interval $(u, v]$. Consider an infinitesimal interval $(t, t+h], h>0$. Then, $\mathbb{P}\left(W_{t}>x ; N_{s}(t, t+\right.$ $h)=1)=\int_{x}^{\infty} \mu r(y) h w^{\mathrm{FIL}}(y) \mathrm{d} y+o(h)$. Note that $\mathbb{P}\left(N_{s}(t, t+h)=1\right) / h($ for $h \rightarrow$ $0)$ is the rate at which customers are taken into service and, since every customer leaves the queue through the server and the system is stable, equals $\lambda$. Combining the above, we have

$$
\begin{aligned}
\mathbb{P}(W>x) & =\lim _{h \rightarrow 0} \mathbb{P}\left(W_{t}>x \mid N_{s}(t, t+h)=1\right) \\
& =\lim _{h \rightarrow 0} \frac{\mathbb{P}\left(W_{t}>x ; N_{s}(t, t+h)=1\right)}{\mathbb{P}\left(N_{s}(t, t+h)=1\right)} \\
& =\frac{1}{\lambda} \int_{x}^{\infty} \mu r(y) w^{\mathrm{FIL}}(y) \mathrm{d} y .
\end{aligned}
$$

The density of the steady-state waiting time, $w(x)$, can be obtained by differentiating the above: 
Corollary 2.1 For the steady-state waiting time, we have $\mathbb{P}(W=0)=W^{\mathrm{FIL}}(0,0)$ and density

$$
w(x)=\frac{\mu r(x) w^{\mathrm{FIL}}(x)}{\lambda},
$$

where $W^{\mathrm{FIL}}(0,0)$ and $w^{\mathrm{FIL}}(\cdot)$ are given in Theorem 2.1 .

Remark 2.1 We note that the steady-state waiting time and FIL distributions take a similar form to the steady-state workload distribution of an M/M/1 queue with workload-dependent arrival and/or service rate; see for example [4, 16] or [2], p. 388. Also related is the elapsed waiting time process in the M/G/1 queue [22].

For positive values, the FIL-process is a special case of the model considered in [6], i.e., an on/off storage system with state-dependent rates restricted to up intervals. Applying [6, Theorem 1] combined with [6, Sect. 6] and taking (in the notation of [6] $r_{0}(x) \equiv 1, \lambda_{0}(x)=\mu r(x)$ and $\lambda_{1}(x) / r_{1}(x) \equiv \lambda$ with $\lambda_{1}(x)$ and $r_{1}(x)$ tending to infinity, directly yields the FIL-density represented in (3). Furthermore, combining results on expected excursion times [6, Theorem 2] with standard renewal arguments provides the remaining constants.

Remark 2.2 For a renewal arrival process, the interior part of the state space can be straightforwardly adapted. In particular, $W_{t}$ is still a Markov process for positive waiting times and the level crossing equation (2) then reads

$$
w^{\mathrm{FIL}}(x)=\int_{y=x}^{\infty} \mu r(y) w^{\mathrm{FIL}}(y)(1-A(y-x)) \mathrm{d} y,
$$

where $A(\cdot)$ is the interarrival-time distribution. Note that the above equation can be written as a Volterra integral equation of the second kind; see for example [28]. For the FIL-process to be a Markov process, a supplementary variable is required to describe the elapsed interarrival time at the boundary of the state space, i.e., in the case there is no customer in line. We note that Corollary 2.1 remains valid for a renewal arrival process.

Example 2.1 The results become even more tractable in various special cases. Here, we consider the case of two service speeds determined by a threshold value of the waiting time of the first customer in the queue. Specifically, we assume that

$$
r(x)= \begin{cases}r_{1}, & \text { for } 0 \leq x \leq K, \\ r_{2}, & \text { for } x>K\end{cases}
$$

This example may serve as an approximation for the case of two heterogeneous servers in Sect. 3, where the secondary server is only activated as soon as the FILprocess exceeds $K$.

Using Theorem 2.1 and Corollary 2.1, we may easily obtain the steady-state distribution of the FIL-process and the waiting time. Here, we present the atom at zero and 


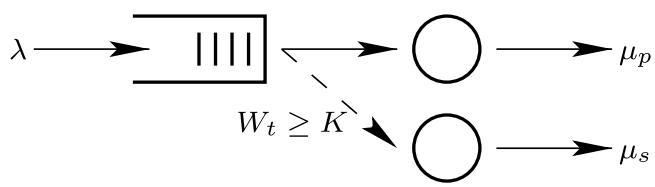

Fig. 1 The queue is served by a primary server with rate $\mu_{p}$ which is supplemented by a secondary server with service rate $\mu_{s}$, when the waiting time of the first in line, $W_{t}$, equals or exceeds $K$

the density of the waiting time. Let $\rho_{i}=\lambda /\left(\mu r_{i}\right)$, for $i=1,2$. After some straightforward calculations, we obtain

$$
w(x)= \begin{cases}r_{1} \mu \rho_{1} W(0) e^{-r_{1} \mu\left(1-\rho_{1}\right) x}, & \text { for } 0<x \leq K, \\ r_{2} \mu \rho_{1} W(0) e^{\left(r_{2}-r_{1}\right) \mu K} e^{-r_{2} \mu\left(1-\rho_{2}\right) x}, & \text { for } x>K,\end{cases}
$$

where

$$
W(0)=\left[\frac{1}{1-\rho_{1}}+\rho_{1} e^{-r_{1} \mu\left(1-\rho_{1}\right) K}\left(\frac{1}{1-\rho_{2}}-\frac{1}{1-\rho_{1}}\right)\right]^{-1} .
$$

\section{Two-server queue}

In this section we turn our attention to a system with two heterogeneous servers. As in Sect. 2 we use the concept of a FIL-process, where $W_{t}$ denotes the waiting time of the first customer in line at time $t$. Again customers arrive to the queue according to a Poisson process with rate $\lambda$. A primary server handles jobs with exponentially distributed service times with mean $1 / \mu_{p}$. A secondary server starts serving customers when $W_{t}$ exceeds a threshold $K$. The service times at the secondary server are exponentially distributed with mean $1 / \mu_{s}$. As in the one-server model of Sect. 2 , the service discipline is FIFO and the servers will always complete a started job, i.e., the secondary server will finish an already started job even if $W_{t}$ drops below $K$ due to a service completion. In this section $Y_{t}$ refers to the number of active secondary servers at time $t$, thus $Y_{t} \in\{0,1\}$. For the system to be stable we assume $\lambda<\mu_{p}+\mu_{s}$. The described two-server system is depicted in Fig. 1.

When dealing with the two-server setup, we introduce the steady-state joint distribution of the FIL-process as $W_{i}^{\mathrm{FIL}}(x)=\lim _{t \rightarrow \infty} \mathbb{P}\left(W_{t} \leq x ; Y_{t}=i\right)$. The joint steadystate density of the FIL-process is denoted $w_{i}^{\mathrm{FIL}}(x)$.

A sample path of the FIL-process is shown in Fig. 2. $W_{t}$ increases linearly with time whenever a customer is in the queue. When the $n$th customer enters service at time $t$, the waiting time of the first in line decreases with $\min \left(A_{n}, W_{t^{-}}\right)$from $W_{t^{-}}$ to $W_{t^{+}}=\max \left(W_{t^{-}}-A_{n}, 0\right)$, where $A_{n}$ is the exponentially distributed interarrival time with rate $\lambda$ between customers $n$ and $n+1$. Because both service times and interarrival times are exponentially distributed, the FIL-process is Markovian.

The analysis of the system is based on the level crossing equations for the FILprocess. These are more involved, compared to those in Sect. 2, and are thus presented in Lemma 3.1. From this, the steady-state distribution of the FIL-process is determined and given in Theorem 3.1. 


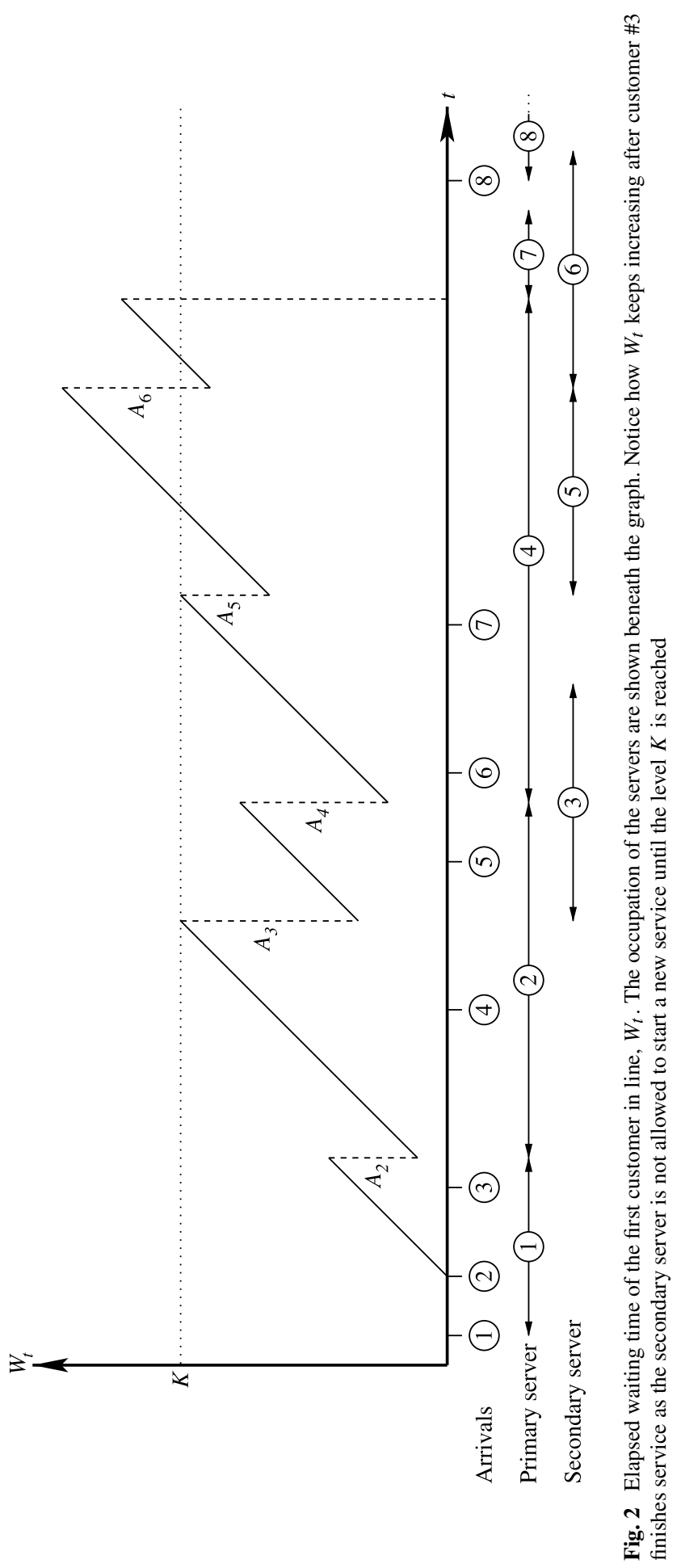


Lemma 3.1 We consider the level crossing equations for three different cases.

(i) For $x<K$ and an active secondary server we have

$$
\begin{aligned}
w_{1}^{\mathrm{FIL}}(x)+\mu_{s} W_{1}^{\mathrm{FIL}}(x)= & \mu_{p} \int_{x}^{\infty} e^{-\lambda(y-x)} w_{1}^{\mathrm{FIL}}(y) \mathrm{d} y \\
& +\mu_{s} \int_{K}^{\infty} e^{-\lambda(y-x)} w_{1}^{\mathrm{FIL}}(y) \mathrm{d} y \\
& +w_{0}^{\mathrm{FIL}}(K-) e^{-\lambda(K-x)} .
\end{aligned}
$$

(ii) For $x<K$ and an inactive secondary server

$$
w_{0}^{\mathrm{FIL}}(x)=\mu_{p} \int_{x}^{K} e^{-\lambda(y-x)} w_{0}^{\mathrm{FIL}}(y) \mathrm{d} y+\mu_{s} W_{1}^{\mathrm{FIL}}(x) .
$$

(iii) For $x>K$ the secondary server will always be active

$$
w_{1}^{\mathrm{FIL}}(x)=\left(\mu_{p}+\mu_{s}\right) \int_{x}^{\infty} e^{-\lambda(y-x)} w_{1}^{\mathrm{FIL}}(y) \mathrm{d} y .
$$

Proof Only case (i) is dealt with in detail as it is the most complicated. The level crossing equations are obtained from setting up forward Kolmogorov equations. For case (i) this becomes

$$
\begin{aligned}
& \mathbb{P}\left(W_{t+h} \leq x+h ; Y_{t+h}=1\right) \\
&=\left(1-\mu_{p} h-\mu_{s} h\right) \mathbb{P}\left(W_{t} \leq x ; Y_{t}=1\right) \\
&+\mu_{p} h \mathbb{P}\left(W_{t} \leq x+A_{n} ; Y_{t}=1\right) \\
&+\mu_{s} h \mathbb{P}\left(K<W_{t} \leq x+A_{n} ; Y_{t}=1\right) \\
&+\left(1-\mu_{p} h\right) \mathbb{P}\left(W_{t} \in[K-h, K] ; W_{t} \leq x+A_{n} ; Y_{t}=0\right)+o(h) .
\end{aligned}
$$

Subtracting $\mathbb{P}\left(W_{t} \leq x+h ; Y_{t}=1\right)$ from both sides, dividing by $h$ and letting $h \rightarrow 0$ allows us to rewrite the term on the left side and the first term on the right side as derivatives with regard to $t$ and $x$ respectively. Moreover $h$ cancels from the rest of the terms except the last. Note that $\mu_{p} \mathbb{P}\left(W_{t} \in[K-h, K] ; W_{t} \leq x+A_{n} ; Y_{t}=0\right) \rightarrow 0$ for $h \rightarrow 0$. Hence,

$$
\begin{aligned}
\frac{\mathrm{d}}{\mathrm{d} t} \mathbb{P}\left(W_{t} \leq x ; Y_{t}=1\right) \\
=-\frac{\mathrm{d}}{\mathrm{d} x} \mathbb{P}\left(W_{t} \leq x ; Y_{t}=1\right)-\left(\mu_{p}+\mu_{s}\right) \mathbb{P}\left(W_{t} \leq x ; Y_{t}=1\right) \\
\quad+\mu_{p} \mathbb{P}\left(W_{t} \leq x+A_{n} ; Y_{t}=1\right)+\mu_{s} \mathbb{P}\left(K<W_{t} \leq x+A_{n} ; Y_{t}=1\right) \\
\quad+\lim _{h \rightarrow 0} \frac{\mathbb{P}\left(W_{t} \leq K ; Y_{t}=0\right)-\mathbb{P}\left(W_{t} \leq K-h ; Y_{t}=0\right)}{h} \cdot \mathbb{P}\left(A_{n}>K-x\right) .
\end{aligned}
$$


By letting $t \rightarrow \infty$, the left side of the expression tends to zero. The probabilities can be written in form of density and distribution functions, using convolution for the probabilities involving $A_{n}$; e.g. $\mathbb{P}\left(W_{t} \leq x+A_{n} ; Y_{t}=1\right)=W_{1}^{\mathrm{FIL}}(x)+\mathbb{P}\left(x<W_{t} \leq\right.$ $\left.x+A_{n}, Y_{t}=1\right)=W_{1}^{\mathrm{FIL}}(x)+\int_{y=x}^{\infty} e^{-\lambda(y-x)} w_{1}^{\mathrm{FIL}}(y) \mathrm{d} y . \quad$ Using $\lim _{h \rightarrow 0, t \rightarrow \infty}\left(\frac{\mathbb{P}\left(W_{t} \leq K ; Y_{t}=0\right)-\mathbb{P}\left(W_{t} \leq K-h ; Y_{t}=0\right)}{h}\right)=w_{0}^{\mathrm{FIL}}(K-)$, then leads to

$$
\begin{aligned}
0= & -w_{1}^{\mathrm{FIL}}(x)-\left(\mu_{p}+\mu_{s}\right) W_{1}^{\mathrm{FIL}}(x) \\
& +\mu_{p}\left(W_{1}^{\mathrm{FIL}}(x)+\int_{x}^{\infty} e^{-\lambda(y-x)} w_{1}^{\mathrm{FIL}}(y) \mathrm{d} y\right)+\mu_{s} \int_{K}^{\infty} e^{-\lambda(y-x)} w_{1}^{\mathrm{FIL}}(y) \mathrm{d} y \\
& +w_{0}^{\mathrm{FIL}}(K-) e^{-\lambda(K-x)} .
\end{aligned}
$$

Finally, the level crossing equation for case (i) can be obtained by simply rearranging the above terms.

We now turn to case (ii). Following an approach similar to the one for case (i), the level crossing equation can be found from the initial Kolmogorov equation

$$
\begin{aligned}
\mathbb{P}\left(W_{t+h} \leq x+h ; Y_{t+h}=0\right)= & \left(1-\mu_{p} h\right) \mathbb{P}\left(W_{t} \leq x ; Y_{t}=0\right) \\
& +\mu_{p} h \mathbb{P}\left(W_{t} \leq x+A_{n} ; Y_{t}=0\right) \\
& +\mu_{s} h \mathbb{P}\left(W_{t} \leq x ; Y_{t}=1\right)+o(h) .
\end{aligned}
$$

In case (iii) the Kolmogorov equation is of the following form:

$$
\begin{aligned}
\mathbb{P}\left(W_{t+h} \leq x+h ; Y_{t+h}=1\right)= & \left(1-\mu_{p} h-\mu_{s} h\right) \mathbb{P}\left(W_{t} \leq x ; Y_{t}=1\right) \\
& +\left(\mu_{p}+\mu_{s}\right) h \mathbb{P}\left(W_{t} \leq x+A_{n} ; Y_{t}=1\right)+o(h) .
\end{aligned}
$$

Again, using the same approach as for case (i), the level crossing equation of Lemma 3.1, case (iii), can be obtained.

Theorem 3.1 The density of the FIL-process, for $Y_{t}=0$, is

$$
w_{0}^{\mathrm{FIL}}(x)=-c_{1} e^{\left(\lambda-\mu_{p}\right) x}-r_{1} c_{3} e^{r_{1} x}-r_{2} c_{4} e^{r_{2} x}, \quad \text { for } 0<x<K,
$$

and, for $Y_{t}=1$, it is

$$
w_{1}^{\mathrm{FIL}}(x)= \begin{cases}r_{1} c_{3} e^{r_{1} x}+r_{2} c_{4} e^{r_{2} x}, & \text { for } 0<x<K \\ c_{2} e^{\left(\lambda-\mu_{p}-\mu_{s}\right) x}, & \text { for } x>K,\end{cases}
$$

with $r_{1}, r_{2}$ given by (6) and (7). The marginal density of the FIL-process for the two-server system becomes

$$
w^{\mathrm{FIL}}(x)= \begin{cases}c_{1} e^{\left(\lambda-\mu_{p}\right) x}, & \text { for } 0<x<K \\ c_{2} e^{\left(\lambda-\mu_{p}-\mu_{s}\right) x}, & \text { for } x>K\end{cases}
$$

The constants $c_{i}, i \in\{1,2,3,4\}$, are determined in Subsect. 3.1. 
Proof The densities of the FIL-process are found from the level crossing equations given in Lemma 3.1. The derivative with respect to $x$ of the level crossing equation in case (i) becomes

$$
\begin{aligned}
w_{1}^{\mathrm{FIL}^{\prime}}(x)+\mu_{s} W_{1}^{\mathrm{FIL}^{\prime}}(x)=\lambda[ & \mu_{p} \int_{x}^{\infty} e^{-\lambda(y-x)} w_{1}^{\mathrm{FIL}}(y) \mathrm{d} y \\
& +\mu_{s} \int_{K}^{\infty} e^{-\lambda(y-x)} w_{1}^{\mathrm{FIL}}(y) \mathrm{d} y \\
& \left.+w_{0}^{\mathrm{FIL}}(K-) e^{-\lambda(K-x)}\right] \\
& -\mu_{p} w_{1}^{\mathrm{FIL}}(x),
\end{aligned}
$$

where the first and last term on the right-hand side of the above equation stem from the derivative of $\mu_{p} \int_{x}^{\infty} e^{-\lambda(y-x)} w_{1}^{\mathrm{FIL}}(y) \mathrm{d} y$. By rearranging and noting that the term inside the brackets equals $w_{1}^{\mathrm{FIL}}(x)+\mu_{s} W_{1}^{\mathrm{FIL}}(x)$, as given in the level crossing equation, we end up with a second-order differential equation:

$$
W_{1}^{\mathrm{FIL}}(x)+\left[\mu_{p}+\mu_{s}-\lambda\right] W_{1}^{\mathrm{FIL}}(x)-\lambda \mu_{s} W_{1}^{\mathrm{FIL}}(x)=0 .
$$

The general solution of (4) is of the form

$$
W_{1}^{\mathrm{FIL}}(x)=c_{3} e^{r_{1} x}+c_{4} e^{r_{2} x},
$$

where

$$
\begin{aligned}
& r_{1}=\frac{\lambda-\left(\mu_{p}+\mu_{s}\right)-\sqrt{\left(\mu_{p}+\mu_{s}-\lambda\right)^{2}+4 \lambda \mu_{s}}}{2}, \\
& r_{2}=\frac{\lambda-\left(\mu_{p}+\mu_{s}\right)+\sqrt{\left(\mu_{p}+\mu_{s}-\lambda\right)^{2}+4 \lambda \mu_{s}}}{2}
\end{aligned}
$$

and $c_{3}$ and $c_{4}$ are constants. The derivative of (5) with respect to $x$ yields the density, $w_{1}^{\mathrm{FIL}}(x)$, for $0<x<K$, as given in Theorem 3.1.

The expressions for $w_{0}^{\mathrm{FIL}}(x)$ for $x<K$ and $w_{1}^{\mathrm{FIL}}(x)$ for $x>K$ can be found in the same way as the solution to the derivative of the level crossing equations in cases (ii) and (iii) of Lemma 3.1 respectively. Finally, the marginal density of $w^{\mathrm{FIL}}(x)$ is found as the sum of $w_{0}^{\mathrm{FIL}}(x)$ and $w_{1}^{\mathrm{FIL}}(x)$.

\subsection{Constants and atoms}

To fully describe the distribution of the FIL-process, the atoms at zero must be determined together with the constants in Theorem 3.1. The atoms, corresponding to the queue being empty, can be divided into four different boundary states; both servers are unoccupied $(\mathrm{N})$, only the primary server is occupied $(\mathrm{P})$, only the secondary server is occupied (S), and both servers are occupied (PS). The probabilities of being 
in these states are referred to as $W_{\mathrm{N}}^{\mathrm{FIL}}(0), W_{\mathrm{P}}^{\mathrm{FIL}}(0), W_{\mathrm{S}}^{\mathrm{FIL}}(0)$ and $W_{\mathrm{PS}}^{\mathrm{FIL}}(0)$, respectively.

Eight independent equations are needed to determine the eight constants; the probability of being in the four boundary states and the $c_{i}$ 's, $i \in\{1,2,3,4\}$. Two equations follow directly from the boundary states in 0 , as $\mathrm{N}$ and $\mathrm{S}$ can only be entered and left from other boundary states. Writing the rate out of the states on the left-hand side and the rate into the states on the right-hand side gives

$$
\lambda W_{\mathrm{N}}^{\mathrm{FIL}}(0)=\mu_{p} W_{\mathrm{P}}^{\mathrm{FIL}}(0)+\mu_{s} W_{\mathrm{S}}^{\mathrm{FIL}}(0)
$$

and

$$
\left(\lambda+\mu_{s}\right) W_{\mathrm{S}}^{\mathrm{FIL}}(0)=\mu_{p} W_{\mathrm{PS}}^{\mathrm{FIL}}(0) .
$$

The rate out of $\mathrm{P}$ is $\lambda+\mu_{p}$ as this state can only be left by an arrival or a departure from the primary server. The state can be entered by an arrival in state $\mathrm{N}$ or a departure from the secondary server in state PS. P can also be entered from the FIL-process for non-zero $W_{t}$ given that $Y_{t}=0$ and $W_{t}<A_{n}$. This is represented by the second term on the right-hand side in (10).

$$
\left(\lambda+\mu_{p}\right) W_{\mathrm{P}}^{\mathrm{FIL}}(0)=\lambda W_{\mathrm{N}}^{\mathrm{FIL}}(0)+\mu_{p} \int_{0^{+}}^{K} e^{-\lambda y} w_{0}^{\mathrm{FIL}}(y) \mathrm{d} y+\mu_{s} W_{\mathrm{PS}}^{\mathrm{FIL}}(0) .
$$

The balance equation for $W_{\mathrm{PS}}^{\mathrm{FIL}}(0)$ is found in the same way:

$$
\begin{aligned}
\left(\lambda+\mu_{p}+\mu_{s}\right) W_{\mathrm{PS}}^{\mathrm{FIL}}(0)= & \lambda W_{\mathrm{S}}^{\mathrm{FIL}}(0)+\mu_{p} \int_{0^{+}}^{\infty} e^{-\lambda y} w_{1}^{\mathrm{FIL}}(y) \mathrm{d} y \\
& +\mu_{s} \int_{K}^{\infty} e^{-\lambda y} w_{1}^{\mathrm{FIL}}(y) \mathrm{d} y+w_{0}^{\mathrm{FIL}}(K-) e^{-\lambda K} .
\end{aligned}
$$

Three more equations can be obtained by considering boundary conditions. By letting $x \downarrow 0$ in (5) we have

$$
W_{\mathrm{S}}^{\mathrm{FIL}}(0)+W_{\mathrm{PS}}^{\mathrm{FIL}}(0)=c_{3}+c_{4} .
$$

Letting $x \uparrow K$ in the level crossing equation of case (ii) in Lemma 3.1 gives

$$
\begin{aligned}
w_{0}^{\mathrm{FIL}}(K-) & =\mu_{s} W_{1}^{\mathrm{FIL}}(K) \\
& =\mu_{s}\left[W_{\mathrm{S}}^{\mathrm{FIL}}(0)+W_{\mathrm{PS}}^{\mathrm{FIL}}(0)+\int_{0}^{K} w_{1}^{\mathrm{FIL}}(y) \mathrm{d} y\right],
\end{aligned}
$$

and the same limit in the level crossing equation of case (i) gives

$$
\begin{aligned}
w_{1}^{\mathrm{FIL}}(K-)+\mu_{s} W_{1}^{\mathrm{FIL}}(K) & =w_{0}^{\mathrm{FIL}}(K-)+\left(\mu_{p}+\mu_{s}\right) \int_{K}^{\infty} e^{-\lambda(y-K)} w_{1}^{\mathrm{FIL}}(y) \mathrm{d} y \\
& =w_{0}^{\mathrm{FIL}}(K-)+c_{2} e^{\left(\lambda-\mu_{p}-\mu_{s}\right) K}
\end{aligned}
$$


The final equation is obtained by normalization of the FIL-process:

$$
\begin{aligned}
1= & \int_{0}^{K} w_{0}^{\mathrm{FIL}}(y) \mathrm{d} y+\int_{0}^{\infty} w_{1}^{\mathrm{FIL}}(y) \mathrm{d} y+W_{\mathrm{N}}^{\mathrm{FI}}(0)+W_{\mathrm{S}}^{\mathrm{FIL}}(0) \\
& +W_{\mathrm{P}}^{\mathrm{FIL}}(0)+W_{\mathrm{PS}}^{\mathrm{FIL}}(0) .
\end{aligned}
$$

The analytical expressions for the constants do not seem to give any additional insight into the problem. Solving the equations numerically is straightforward. We have shown that at most two of the equations can be mutually dependent and all numerical investigations point toward them being independent. Furthermore we argue that as long as the requirements for stability of the system are fulfilled, a unique solution to the equation array must exist and thus the equations must indeed be independent.

\subsection{Waiting-time distribution}

We now turn to the waiting-time distribution and use the same definition of this as in Sect. $2 ; W(x)=\mathbb{P}(W \leq x)$, where $W$ is the waiting time an arbitrary customer experiences. Observe that arriving customers are directly taken into service in case the queue is empty and the primary server is available. Using PASTA, it is easy to obtain the atom at zero of the waiting time:

$$
\mathbb{P}(W=0)=W_{\mathrm{N}}^{\mathrm{FIL}}(0)+W_{\mathrm{S}}^{\mathrm{FIL}}(0) .
$$

In case the waiting time is non-zero, the waiting time corresponds to the FILprocess at epochs right before downward jumps. Here, we again consider an infinitesimal interval $(t, t+h)$ and apply similar arguments as in Sect. 2. In particular, for $x \geq K$, we have

$$
\mathbb{P}\left(W_{t}>x ; N_{s}(t, t+h)=1\right)=\left(\mu_{p}+\mu_{s}\right) h \int_{x}^{\infty} w_{1}^{\mathrm{FIL}}(y) \mathrm{d} y+o(h) .
$$

For $0<x<K$, we have

$$
\begin{aligned}
\mathbb{P}\left(W_{t}>x ; N_{s}(t, t+h)=1\right)= & \mu_{p} h \int_{x}^{K-h} w_{0}^{\mathrm{FIL}}(y) \mathrm{d} y+\mu_{p} h \int_{x}^{K} w_{1}^{\mathrm{FIL}}(y) \mathrm{d} y \\
& +\int_{K-h}^{K} w_{0}^{\mathrm{FIL}}(y) \mathrm{d} y \\
& +\left(\mu_{p}+\mu_{s}\right) h \int_{K}^{\infty} w_{1}^{\mathrm{FIL}}(y) \mathrm{d} y+o(h) .
\end{aligned}
$$

Note that $\int_{K-h}^{K} w_{0}^{\mathrm{FIL}}(y) \mathrm{d} y / h \rightarrow w_{0}^{\mathrm{FIL}}(K-)$, as $h \rightarrow 0$. Also, observe that $\mathbb{P}\left(N_{s}(t, t+\right.$ $h)=1) / h$ (for $h \rightarrow 0$ ) is the rate at which customers are taken into service and, since every customer leaves the queue through the server and the system is stable, equals 
$\lambda$. Combining the above and using a similar conditioning as in Sect. 2, we obtain

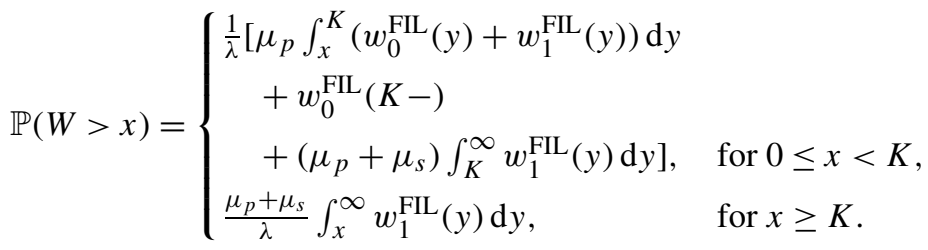

From this, we obtain the density of the steady-state waiting time and the atom at $K$ :

Corollary 3.1 For the steady-state waiting time, we have two atoms

$$
\begin{aligned}
& \mathbb{P}(W=0)=W_{\mathrm{N}}^{\mathrm{FIL}}(0)+W_{\mathrm{S}}^{\mathrm{FIL}}(0), \\
& \mathbb{P}(W=K)=\frac{w_{0}^{\mathrm{FIL}}(K-)}{\lambda},
\end{aligned}
$$

and density

$$
w(x)= \begin{cases}\frac{\mu_{p}}{\lambda} c_{1} e^{\left(\lambda-\mu_{p}\right) x}, & \text { for } 0<x<K, \\ \frac{\mu_{p}+\mu_{s}}{\lambda} c_{2} e^{\left(\lambda-\mu_{p}-\mu_{s}\right) x}, & \text { for } x>K .\end{cases}
$$

Remark 3.1 Note that the form of the steady-state waiting time density (and distribution) is closely related to the density in Example 2.1, i.e., the single-server model with two service speeds determined by a threshold on the FIL-process. In particular, the parameters $r_{i}, i=1,2$, and $\mu$ should be taken such that $r_{1} \mu=\mu_{p}$ and $r_{2} \mu=\mu_{p}+\mu_{s}$ (for instance, let $\mu=\mu_{p}, r_{1}=1$, and $r_{2}=1+\mu_{s} / \mu_{p}$ ). The main difference between the waiting-time distributions concerns the atom at $K$.

\section{Numerical results}

To illustrate the difference in behavior of the waiting-time distribution for the oneserver system of Example 2.1 and the two-server system treated in Sect. 3, a few numerical results are shown in Fig. 3. The parameters have been chosen such that the two cases are comparable.

The waiting-time distributions in Fig. 4 are found from Corollary 3.1 and the corresponding eight constants, found with Maple, are given in Table 1. It is seen how the relation between $\lambda$ and $\mu_{p}$ governs the shape of the distribution for $x<K$; it is convex for $\lambda<\mu_{p}$, concave for $\lambda>\mu_{p}$ and a straight line for $\lambda=\mu_{p}$. Notable are also the atoms at $K$ which are absent in the two-speed single-server case of Fig. 4.

The somewhat better performance of the two-server model can be explained by the secondary server finishing an already started service when $W_{t}$ drops below $K$, whereas the single-server system of Example 2.1 will change the service speed to $r_{1}$ immediately.

In Fig. 4 we compared the performance of the service mechanism based on waiting times to the control based on queue lengths, since the latter is common in the queueing literature. For the model with queue-length based control, the secondary server is 


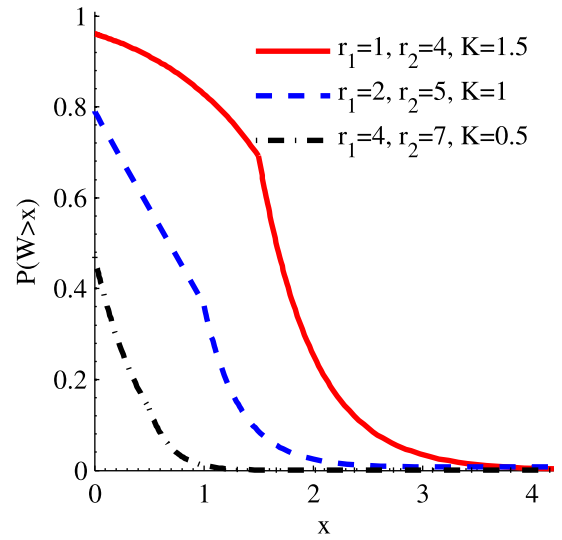

(a) Waiting-time distributions for the single server system in Example 2.1 with two service speeds determined by different parameter values of $r_{1}, r_{2}$, and $K$. Shared parameters: $\lambda=2$ and $\mu=1$.

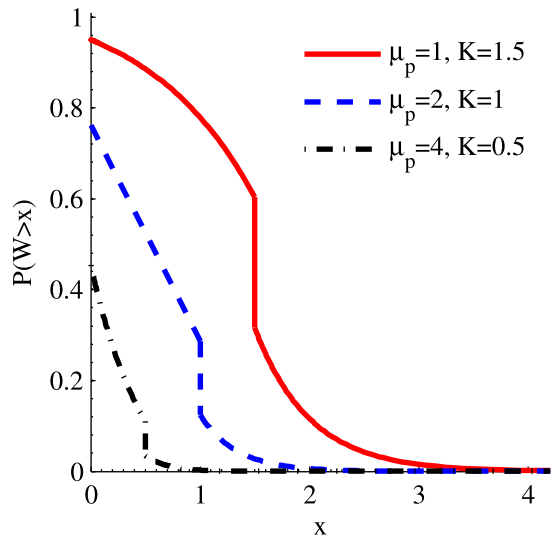

(b) Waiting-time distribution for the two-server system of Section 3 for 3 different values of $\mu_{p}$ and $K$ and shared parameters $\lambda=2$ and $\mu_{s}=3$.

Fig. 3 Numerical comparison of the one- and two-server models

Table 1 Numerical results for common parameters $\lambda=2$, $\mu_{s}=3$

\begin{tabular}{llll}
\hline & $\left(\mu_{p}=1, K=1.5\right)$ & $\left(\mu_{p}=2, K=1.0\right)$ & $\left(\mu_{p}=4, K=0.5\right)$ \\
\hline$W_{\mathrm{N}}$ & 0.0470 & 0.2298 & 0.5318 \\
$W_{\mathrm{P}}$ & 0.0860 & 0.2181 & 0.2559 \\
$W_{\mathrm{S}}$ & 0.0027 & 0.0078 & 0.0133 \\
$W_{\mathrm{PS}}$ & 0.0135 & 0.0195 & 0.0166 \\
$c_{1}$ & 0.1990 & 0.4751 & 0.5451 \\
$c_{2}$ & 6.3453 & 2.9956 & 0.6123 \\
$c_{3}$ & $-0.6401 \cdot 10^{-4}$ & $-0.2673 \cdot 10^{-3}$ & $-0.4749 \cdot 10^{-3}$ \\
$c_{4}$ & 0.01626 & 0.0276 & 0.0304 \\
\hline
\end{tabular}

only allowed to take customers into service when more than 30 and three customers, in Figs. 4a and 4b, respectively, are waiting in the queue. These parameters have been chosen such that the resulting average waiting times are nearly identical for the two policies. The waiting-time distribution for the queue-length based threshold is found by taking the average of 50 simulations of 100,000 calls each. In this way the $95 \%$ confidence intervals become too narrow to display in the figure. It is seen that the waiting-time based threshold results in less variation of waiting times which is preferable as the objective is to have more control over the system. This reduction in variability of waiting times is accentuated for larger threshold value as displayed in Fig. 4. The figure illustrates the interesting, but not surprising, phenomenon of how the probability mass gathers around $K$ for $\lambda>\mu_{p}, K$ large and waiting-time based control.

Given the distribution of the waiting-time and FIL-process, most of the commonly used performance measures such as TSF are easily found. Other performance mea- 


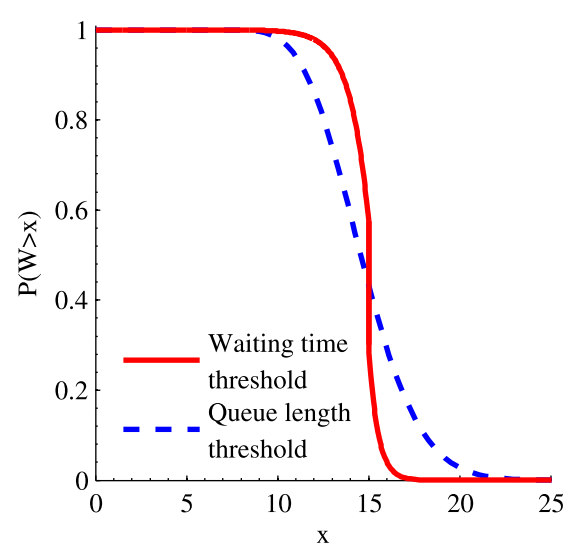

(a) Waiting-time distribution for large $K,(\lambda=2$, $\left.\mu_{p}=1, \mu_{s}=3, K=15\right)$ compared to a similar setup with queue-length based threshold of 30 .

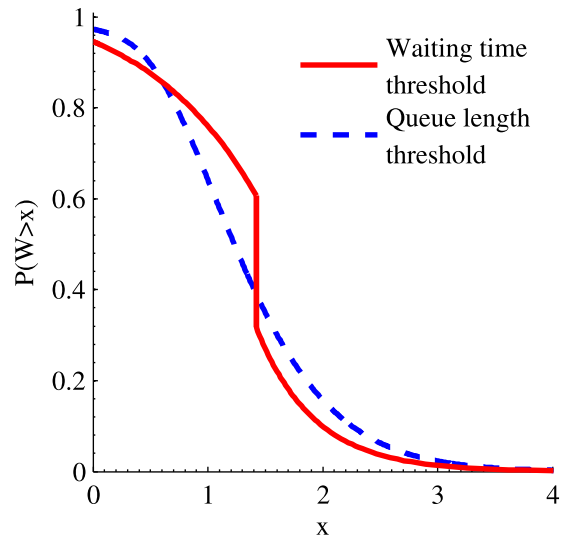

(b) Waiting-time distribution for small $K,(\lambda=2$, $\left.\mu_{p}=1, \mu_{s}=3, K=1.42\right)$ compared to a similar setup with queue-length based threshold of 3 .

Fig. 4 Waiting-time thresholds compared to queue-length thresholds

sures such as the utilization of the servers can be found as

$$
\begin{aligned}
& a_{p}=1-W_{\mathrm{N}}(0)-W_{\mathrm{S}}(0), \\
& a_{s}=1-W_{\mathrm{N}}(0)-W_{\mathrm{P}}(0)-\int_{0}^{K} w_{0}^{\mathrm{FIL}}(y) \mathrm{d} y,
\end{aligned}
$$

where $a_{p}$ and $a_{s}$ are the utilization of the primary and secondary server, respectively.

\section{Conclusions and topics for further research}

We have studied queueing systems where the provided service depends on the waiting time of the first customer in line. This type of control is commonly used in call centers and has mainly been motivated by a frequently used setup referred to as an "inverted V"; see [1]. The main contribution is that we have shown ways to deal with systems where the service changes depending on the waiting time, which can be inherently difficult to deal with in particular in the case of fixed thresholds.

The first model of this paper deals with a single server that operates with a service speed depending on the waiting time of the first customer in line. We derived the waiting-time distribution of an arbitrary customer entering the system and showed how the model can be used for the threshold case.

The second model of this paper deals with a two-server setup where a secondary server supplements a primary server when the waiting time of the first in line exceeds a threshold. Again the waiting distribution of an arbitrary customer has been derived and numerical examples have been given. It was illustrated that a waiting-time based threshold is preferable to a queue-length based, when a high degree of control of the waiting times is desired. Also, the simplicity of the form of the solution for the waiting time given in Corollary 3.1 provides some useful insight. 
In the model presented in Sect. 3, only one primary and one secondary server was considered. This is easily extended to a more general setup with multiple primary servers by introducing additional states for $W^{\mathrm{FIL}}(0)$ along with the four already used. The extra boundary states should describe the number of unoccupied servers. Analyzing a setup with multiple secondary servers would be much more difficult as the joint distribution of $w_{i}^{\mathrm{FIL}}(x)$ must be extended to include $i \in\{0,1, \ldots, n\}$, where $n$ is the number of secondary servers.

A related routing setup, often seen in call centers and used as a way to prioritize a group of customers over another, is the "N" design, see [14]. Also related are [12, 13] and [15]. The "N" design is basically an extension to the model of Sect. 3 where the secondary server also has a queue of its own, from which it receives jobs. Extending the model presented in this paper to the "N" design, necessitates the use of a 2-dimensional FIL-process in order to keep track of the waiting time of the first customer in line in both queues.

There is still much to be done in relation to analysis of complex queueing systems such as those seen in call centers. Even though simulation may remain the dominant way of modeling these systems, it is indeed worth pursuing analytical approaches to gain insight not obtainable through simulation such as the result in Corollary 3.1.

Open Access This article is distributed under the terms of the Creative Commons Attribution Noncommercial License which permits any noncommercial use, distribution, and reproduction in any medium, provided the original author(s) and source are credited.

\section{References}

1. Armony, M.: Dynamic routing in large-scale service systems with heterogeneous servers. Queueing Syst. 51, 287-329 (2005)

2. Asmussen, S.: Applied Probability and Queues, 2nd edn. Springer, New York (2003)

3. Barth, W., Manitz, M., Stolletz, R.: Analysis of two-level support systems with time-dependent overflow—a banking application. Prod. Oper. Manag. 19, 757-768 (2010)

4. Bekker, R., Borst, S.C., Boxma, O.J., Kella, O.: Queues with workload-dependent arrival and service rates. Queueing Syst. 46, 537-556 (2004)

5. Boxma, O.J., Vlasiou, M.: On queues with service and interarrival times depending on waiting times. Queueing Syst. 56, 121-132 (2007)

6. Boxma, O., Kaspi, H., Kella, O., Perry, D.: On/off storage systems with state dependent input, output and switching rates. Probab. Eng. Inf. Sci. 19, 1-14 (2005)

7. Brill, P.H., Posner, M.J.M.: A two server queue with nonwaiting customers receiving specialized service. Manag. Sci. 27, 914-925 (1981)

8. Brill, P.H., Posner, M.J.M.: The system point method in exponential queues: a level crossing approach. Math. Oper. Res. 6, 31-49 (1981)

9. Browne, S., Sigman, K.: Work-modulated queues with applications to storage processes. J. Appl. Probab. 29, 699-712 (1992)

10. Cohen, J.W., Rubinovitch, M.: On level crossings and cycles in dam processes. Math. Oper. Res. 2, 297-310 (1977)

11. Franx, G.J., Koole, G.M., Pot, S.A.: Approximating multi-skill blocking systems by hyperexponential decomposition. Perform. Eval. 63, 799-824 (2006)

12. Gans, N., Zhou, Y.-P.: A call-routing problem with service-level constraints. Oper. Res. 51, 255-271 (2003)

13. Gans, N., Zhou, Y.-P.: Call-routing schemes for call-center outsourcing. Manuf. Serv. Oper. Manag. 9, 33-50 (2007)

14. Gans, N., Koole, G.M., Mandelbaum, A.: Telephone call centers: tutorial, review, and research prospects. Manuf. Serv. Oper. Manag. 5, 79-141 (2003) 
15. Gurvich, I., Armony, M., Mandelbaum, A.: Service-level differentiation in call centers with fully flexible servers. Manag. Sci. 54, 279-294 (2008)

16. Harrison, J.M., Resnick, S.I.: The stationary distribution and first exit probabilities of a storage process with general release rule. Math. Oper. Res. 1, 347-358 (1976)

17. Jackson, J.R.: Some problems in queueing with dynamic priorities. Nav. Res. Logist. Q. 7, 235-249 (1960)

18. Koole, G.M.: A simple proof of the optimality of a threshold policy in a two-server queueing system. Syst. Control Lett. 26, 301-303 (1995)

19. Lin, W., Kumar, P.R.: Optimal control of a queueing system with two heterogeneous servers. IEEE Trans. Autom. Control 29, 696-703 (1984)

20. Liu, L., Parlar, M., Zhu, S.: Pricing and lead time decisions in decentralized supply chains. Manag. Sci. 53, 713-725 (2007)

21. Lucent Technologies CentreVu Release 8 Advocate User Guide. P.O. Box 4100, Crawfordsville, IN 47933, USA: Lucent Technologies (1999).

22. Perry, D., Benny, L.: Continuous production/inventory model with analogy to certain queueing and Dam models. Adv. Appl. Probab. 21, 123-141 (1989)

23. Posner, M.J.M.: Single-server queues with service time dependent on waiting time. Oper. Res. 21, 610-616 (1973)

24. Rubinovitch, M.: The slow server problem. J. Appl. Probab. 22, 205-213 (1985)

25. Scheinhardt, W.R.W., van Foreest, N., Mandjes, M.: Continuous feedback fluid queues. Oper. Res. Lett. 33, 551-559 (2005)

26. Stockbridge, R.H.: A martingale approach to the slow server problem. J. Appl. Probab. 28, 480-486 (1991)

27. Whitt, W.: Queues with service times and interarrival times depending linearly and randomly upon waiting times. Queueing Syst. 6, 335-351 (1990)

28. Zabreiko, P.P., Koshelev, A.I., Krasnosel'skii, M.A.: Integral Equations: A Reference Text. Monographs and Textbooks on Pure and Applied Mathematics. Noordhoff, Leiden (1975). Transl. and ed. by T.O. Shaposhnikova, R.S. Anderssen and S.G. Mikhlin 